\title{
A Kinematic Survey of Carbon Stars in the Small Magellanic Cloud
}

\section{RUSSELL CANNON ${ }^{1}$, BARRY CROKE ${ }^{2}$, DESPINA HATZIDIMITRIOU ${ }^{2}$, and DAVID MORGAN ${ }^{3}$}

1 Anglo-Australian Observatory, Sydney, Australia

2 University of Crete, Heraklion, Greece

${ }^{3}$ Royal Observatory, Edinburgh, Scotland

We are carrying out a survey of carbon stars in the outer parts of the Small Magellanic Cloud (SMC), using the double-beam spectrograph on the ANU 2.3-m telescope at Siding Spring Observatory. These stars can be used as tracers of the older stellar populations (i.e. with ages of a few billion years). The stars were chosen from the large sample of new carbon stars found by Morgan \& Hatzidimitriou (1995, A $\mathcal{E}$ A Supp., 113, 539) on objective prism photographs taken with the UK 1.2-m Schmidt Telescope. The blue spectra cover the $\mathrm{C}_{2}$ Swan bands and confirm that virtually all the stars are indeed carbon stars, while the higher dispersion red spectra yield velocities accurate to better than $5 \mathrm{~km} \mathrm{~s}^{-1}$.

For 71 carbon stars surrounding the SMC, at radii of between $3^{\circ}$ and $6^{\circ}$, we obtain a mean radial velocity of $146 \pm 3 \mathrm{~km} \mathrm{~s}^{-1}$ and a dispersion of $21 \pm 2 \mathrm{~km} \mathrm{~s}^{-1}$, similar to that found in other intermediate-age and old populations in the SMC. This implies a mass of about $1.2 \times 10^{9}$ solar masses under the somewhat unrealistic assumption of a spherical SMC halo. There is no evidence for any bifurcation of radial velocities as seen in some samples of younger objects, nor are there any significant velocity trends across the face of the SMC.

Three carbon stars have been found mid-way between the two Clouds; their velocities are typical of those in the main SMC sample. For a small sample of 16 stars in the SW corner of the LMC we obtain a higher mean velocity of $226 \pm 3 \mathrm{~km} \mathrm{~s}^{-1}$ with a dispersion of $12 \pm 2 \mathrm{~km} \mathrm{~s}^{-1}$.

A full account of this work has been published by Hatzidimitriou et al. (1997, A \& A Supp., 122, 507). 